\title{
PHASES differential astrometry and the mutual inclination of the V819 Herculis triple star system
}

\author{
M. W. Muterspaugh ${ }^{1,2}$, B. F. Lane ${ }^{1}$, M. Konacki ${ }^{2,3}$, B. F. Burke ${ }^{1}$, M. M. Colavita ${ }^{4}$, S. R. Kulkarni ${ }^{5}$, and M. Shao ${ }^{4}$ \\ 1 MIT Kavli Institute for Astrophysics and Space Research, MIT Department of Physics, 70 Vassar Street, Cambridge, MA 02139, USA \\ e-mail: [matthew1; blane] @mit.edu \\ 2 Department of Geological and Planetary Sciences, California Institute of Technology, MS 150-21, Pasadena, CA 91125, USA \\ e-mail: maciej@gps.caltech.edu \\ 3 Nicolaus Copernicus Astronomical Center, Polish Academy of Sciences, Rabianska 8, 87-100 Torun, Poland \\ 4 Jet Propulsion Laboratory, California Institute of Technology, 4800 Oak Grove Dr., Pasadena, CA 91109, USA \\ 5 Division of Physics, Mathematics and Astronomy, 105-24, California Institute of Technology, Pasadena, CA 91125, USA
}

Received 3 July 2005 / Accepted 19 September 2005

\section{ABSTRACT}

V819 Herculis is a well-studied triple star system consisting of a "wide" pair with 5.5 year period, one component of which is a 2.2-day period eclipsing single-line spectroscopic binary. Differential astrometry measurements from the Palomar High-precision Astrometric Search for Exoplanet Systems (PHASES) are presented and used to determine a relative inclination between the short- and long-period orbits of 23.6 \pm 4.9 degrees. This represents only the sixth unambiguous determination of the mutual inclination of orbits in a hierarchical triple system. This result is combined with those for the other five systems for analysis of the observed distribution of mutual inclinations in nearby triple systems. It is found that this distribution is different than that which one would expect from random orientations with statistical significance at the 94\% level; implications for studying the spatial distribution of angular momentum in star forming regions is discussed.

Key words. stars: individual: V819 Herculis - binaries: close - techniques: interferometric - astrometry

\section{Introduction}

Determinations of the mutual inclinations of the two orbits in hierarchical triple stellar systems are rare, with previously only four unambiguous determinations available in the literature (Lestrade et al. 1993; Heintz 1996; Hummel et al. 2003), but valuable; the dynamical relaxation process undergone by multiples after formation is expected to leave a statistical "fingerprint" in the distribution of inclinations (Sterzik \& Tokovinin 2002). In addition, well-characterized stellar multiples represent excellent opportunities to test and challenge stellar models under stringent constraints of common age and metallicity.

V819 Herculis (HR 6469, HD 157482; $V=5.6, K=4.1$ ) is a triple system consisting of an evolved star (G7 III-IV; this appears brighter in $V$ and will be referred to as the $A$ component) in an eccentric 5.5 year orbit together with a close $\left(P_{n} \approx 2.2\right.$ days $)$ pair of main sequence $\mathrm{F}$ stars. The close pair $(\mathrm{Ba}$ and $\mathrm{Bb})$ is in an edge-on orbit and exhibits shallow eclipses. A combination of radial velocity, speckle interferometry, eclipse timing and light-curve fitting has made it possible to accurately determine most of the interesting system parameters, including masses, radii and distance (Scarfe et al. 1994; van Hamme et al. 1994; Wasson et al. 1994) with accuracies of a few percent. However, until now it has not been possible to determine the mutual inclination of the orbits. The V819 Her system is listed as a chromospherically active binary in the catalog by Strassmeier et al. (1993); it exhibits Ca H and K emission and has been detected in X-rays (Dempsey et al. 1993) but not in radio (Drake et al. 1989). The system is variable with an amplitude of approximately 80 milli-magnitudes. In addition to the eclipses of the close pair, the GIV component exhibits quasi-periodic variability attributed to starspots (van Hamme et al. 1994).

A technique has been developed to obtain high precision (10-20 $\mu$ as) astrometry of close stellar pairs (separation less than one arcsecond; Lane \& Muterspaugh 2004) using long-baseline infrared interferometry at the Palomar Testbed Interferometer (PTI; Colavita et al. 1999). This level of precision allows us to determine the orbital parameters of the V819 Her system at the level of $\sim 3-5 \%$, primarily limited by the quality of available radial velocities. However, because one can measure the astrometric motion of the close pair in the system, it is possible to directly determine the relative inclination of the orbits. These measurements were made as part of the Palomar High-precision Astrometric Search for Exoplanet Systems (PHASES) program. PTI is located 
on Palomar Mountain near San Diego, CA. It was developed by the Jet Propulsion Laboratory, California Institute of Technology for NASA, as a testbed for interferometric techniques applicable to the Keck Interferometer and other missions such as the Space Interferometry Mission, SIM. It operates in the $J(1.2 \mu \mathrm{m}), H(1.6 \mu \mathrm{m})$, and $K(2.2 \mu \mathrm{m})$ bands, and combines starlight from two out of three available 40-cm apertures. The apertures form a triangle with one 110 and two $87 \mathrm{~m}$ baselines.

\section{Observations and data processing}

\subsection{PHASES observations}

V819 Herculis was observed using PTI on 31 nights in 2003-2005 using the observing mode described in Lane \& Muterspaugh (2004). This method for phase-referenced differential astrometry of subarcsecond binaries is briefly reviewed here.

In an optical interferometer light is collected at two or more apertures and brought to a central location where the beams are combined and a fringe pattern produced. For a broadband source of central wavelength $\lambda$ the fringe pattern appears only when the optical paths through the arms of the interferometer are equalized to within a coherence length $\left(\Lambda=\lambda^{2} / \Delta \lambda\right)$. For a two-aperture interferometer, neglecting dispersion, the intensity measured at one of the combined beams is given by

$$
I(x)=I_{0}\left(1+V \frac{\sin (\pi x / \Lambda)}{\pi x / \Lambda} \sin (2 \pi x / \lambda)\right)
$$

where $x$ is the differential amount of path between arms of the interferometer, $V$ is the fringe contrast or "visibility", which can be related to the morphology of the source, and $\Delta \lambda$ is the optical bandwidth of the interferometer assuming a flat optical bandpass (for PTI $\Delta \lambda=0.4 \mu \mathrm{m}$ ).

The location of the resulting interference fringes are related to the position of the target star and the observing geometry via

$d=\vec{B} \cdot \vec{S}+\delta_{a}(\vec{S}, t)+c$

where $d$ is the optical path-length one must introduce between the two arms of the interferometer to find fringes. This quantity is often called the "delay." $\vec{B}$ is the baseline, the vector connecting the two apertures. $\vec{S}$ is the unit vector in the source direction, and $c$ is a constant additional scalar delay introduced by the instrument. The term $\delta_{a}(\vec{S}, t)$ is related to the differential amount of path introduced by the atmosphere over each telescope due to variations in refractive index. For a 100-m baseline interferometer an astrometric precision of $10 \mu$ as corresponds to knowing $d$ to $5 \mathrm{~nm}$, a difficult but not impossible proposition for all terms except that related to the atmospheric delay. Atmospheric turbulence, which changes over distances of tens of centimeters, angles on order tens of arcseconds, and on subsecond timescales, forces one to use very short exposures (to maintain fringe contrast) and hence limits the sensitivity of the instrument. It also severely limits the astrometric accuracy of a simple interferometer, at least over large sky-angles.
However, in narrow-angle astrometry one is concerned with a close pair of stars, and the observable is a differential astrometric measurement, i.e. one is interested in knowing the angle between the two stars $\left(\overrightarrow{\Delta_{s}}=\overrightarrow{s_{2}}-\overrightarrow{s_{1}}\right)$. The atmospheric turbulence is correlated over small angles. If the measurements of the two stars are simultaneous, or nearly so, the atmospheric term subtracts out. Hence it is still possible to obtain high precision "narrow-angle" astrometry (Colavita 1994).

To correct for time-dependent fluctuations in the atmospheric turbulence, observations consisted of operating PTI in a phase-referenced observing mode. After movable mirrors in the beam-combining lab apply delay compensation to the light collected from two $40 \mathrm{~cm}$ apertures, the light from each aperture is split using 30/70 beamsplitters. Seventy percent of the light is sent to the phase-tracking "primary" interferometric beam combiner which measures the time-dependent phase of one star's interferogram (fringes) caused by the atmospheric turbulence, and used in a feed-back loop to control the optical delay lines.

The other $30 \%$ of the light is diverted to the "secondary" interferometric beam combiner. In this system there is an additional delay line with a travel of only $\approx 500$ microns. This is used to introduce delay with a sawtooth waveform with frequency on order a Hertz. This allows us to sample the interferograms of all stars in the one arcsecond detector field whose projected separations are within the scan range. Laser metrology is used along all starlight paths between the 30/70 split and the point of interferometric combination to monitor internal vibrations differential to the phase-referencing and scanning beam combiners. For V819 Herculis, the typical scanning rate in 2003 was one scan per second and four intensity measurements per ten milliseconds; these values were doubled in 2004. The typical scan amplitude was 100 microns. An average of 3099 scans were collected each night the star was observed over a time span of 18 to $179 \mathrm{~min}$.

\subsection{PHASES data reduction}

The data reduction algorithm used was similar to that described in Lane \& Muterspaugh (2004), but now all astrometric fitting is analyzed with differential right ascension and declination as parameters, rather than projected separation. First, detector calibrations (gain, bias, and background) are applied to the intensity measurements. Next, a grid is constructed in differential right ascension and declination over which to search (in ICRS 2000.0 coordinates). For each point in the search grid we calculate the expected differential delay based on the interferometer location, baseline geometry, and time of observation for each scan. These conversions were simplified using the routines from the Naval Observatory Vector Astrometry Subroutines C Language Version 2.0 (NOVAS-C; see Kaplan et al. 1989). A model of a double-fringe packet is then calculated and compared to the observed scan to derive a $\chi^{2}$ value as a merit of goodness-of-fit; this is repeated for each scan, co-adding all of the $\chi^{2}$ values associated with that point in the search grid. The model fringe template is found by observing single stars, incoherently averaging periodograms of their 
interferograms, and fitting a sum of Gaussians to the average periodogram. This model effective bandpass is Fourier transformed into delay space to create a model interferogram. Sample data sets have been reanalyzed with a variety of model interferograms and the resulting astrometric solutions vary by less than one $\mu$ as; this is largely due to the differential nature of the measurement. Note that in addition to the differential delay there are several additional parameters to the double fringe packet: fringe contrast and relative intensities as well as mean delay. These are all adjusted to minimize $\chi^{2}$ on a scan-by-scan basis. The final $\chi^{2}$ surface as a function of differential right ascension and declination is thus derived. The best-fit astrometric position is found at the minimum $\chi^{2}$ position, with uncertainties defined by the appropriate $\chi^{2}$ contour-which depends on the number of degrees of freedom in the problem and the value of the $\chi^{2}$-minimum.

One potential complication with fitting a fringe to the data is that there are many local minima spaced at multiples of the operating wavelength. If one were to fit a fringe model to each scan separately and average (or fit an astrometric model to) the resulting delays, one would be severely limited by this fringe ambiguity (for a $110-\mathrm{m}$ baseline interferometer operating at $2.2 \mu \mathrm{m}$, the resulting positional ambiguity is $\sim 4.1$ milli-arcseconds). However, by using the $\chi^{2}$-surface approach, and co-adding the probabilities associated with all possible delays for each scan, the ambiguity disappears. This is due to two things, the first being that co-adding simply improves the signal-to-noise ratio. Second, since the observations usually last for an hour or even longer, the associated baseline change due to Earth rotation also has the effect of "smearing" out all but the true global minimum. The final $\chi^{2}$-surface does have dips separated by $\sim 4.1$ milli-arcseconds from the true location, but any data sets for which these show up at or above the $4 \sigma$ level are rejected. The final astrometry measurement and related uncertainties are derived by fitting only the $4 \sigma$ region of the surface.

The PHASES data reduction algorithm naturally accounts for contributions from photon and read-noise. Unmonitored phase noise shows up by increasing the minimum value of $\chi^{2}$ surface. Comparison of this value with that expected from the number of degrees of freedom allows us to co-add the phase noise to the fit uncertainties.

This method has been rigorously tested on both synthetic and real data. Data sets are divided into equal sized subsets which are analyzed separately. A Kolmogorov-Smirnov test shows the formal uncertainties from the PHASES data reduction pipeline to be consistent with the scatter between subsets. After an astrometric solution has been determined, one can revisit the individual scans and determine best-fit delay separations on a scan-by-scan basis (the fringe ambiguity now being removed). The differential delay residuals show normal (Gaussian) distribution, and Allan variances of delay residuals agree with the performance levels of the formal uncertainties and show the residuals to be uncorrelated. It is concluded that the PHASES data reduction pipeline produces measurement uncertainties that are consistent with the internal scatter of the data on intra-night timescales.

\subsection{PHASES astrometric results}

The differential astrometry measurements are listed in Table 1, in the ICRS 2000.0 reference frame. In order to evaluate the night-to-night astrometric repeatability of the data, the PHASES data were fit to a model consisting of a Keplerian orbit representing the $\mathrm{Ba}-\mathrm{Bb}$ center of light (CL) motion and a low-order polynomial representing motion of the A-B orbit. The minimized value of reduced $\chi_{r}^{2}=4$, implying either that the internal (i.e. derived from a single night of data) uncertainty estimates are too low by a factor of 2 , or that the simple model is not appropriate for this system. Replacing the polynomial model for A-B with a Keplerian does not improve the value of $\chi_{r}^{2}$ (to be expected given the limited fraction of the A-B orbit covered by the PHASES data set). It is possible starspots caused astrometric jitter on this scale. The PHASES uncertainties presented in this paper have been increased by a factor of 2 to account for this discrepancy. The rescaled (raw) median minor- and major-axis uncertainties are 15.2 (7.6) and 363 (181) $\mu$ as. The rescaled (raw) mean minor- and majoraxis uncertainties are $19.6(9.8)$ and 568 (284) $\mu$ as respectively.

\subsection{Potential systematic errors}

The fractional precision of the PHASES astrometric measurements is $\sim 10^{-4}$; at such an ambitious level there are many possible sources of systematic error that could appear on internight timescales. In particular, the system in question exhibits two potential astrophysical sources of measurement noise: starspots and eclipses.

\subsubsection{Starspots}

The $\approx 40$ milli-magnitude variability of V819 Herculis A has been attributed to starspots. Upper limits to shifts in the CL of a star caused by star spots are evaluated with a model comprised of a uniform stellar disk (radius $R$ ) except for a zerotemperature (non-emitting) circular region of radius $r$ tangent to the edge of the stellar disk (i.e. centered at $x=R-r, y=0$ ). The CL is displaced by:

$$
\begin{aligned}
\frac{x_{c}}{R} & =\frac{\int_{-R}^{R} \int_{-\sqrt{R^{2}-x^{2}}}^{\sqrt{R^{2}-x^{2}}} x \mathrm{~d} y \mathrm{~d} x-\int_{R-2 r}^{R} \int_{-\sqrt{r^{2}-(x-R+r)^{2}}}^{\sqrt{r^{2}-(x-R+r)^{2}}} x \mathrm{~d} y \mathrm{~d} x}{R \pi\left(R^{2}-r^{2}\right)} \\
& =-\frac{r^{2} / R^{2}}{1+r / R} .
\end{aligned}
$$

The non-emitting spots in this model would cause photometric variations proportional to the fractional area of the stellar disk covered:

$\frac{F}{F_{o}}=1-\frac{r^{2}}{R^{2}}$

where $F_{o}$ is the star's flux when no spots are present. Equations (3) and (4) provide a relationship between the apparent astrometric and photometric shifts caused by star spots. For stars of typical radius 1 milli-arcsecond, the simplified model gives a roughly linear relationship of $0.8 \mu$ as of maximum astrometric shift per milli-magnitude of photometric variability. 
Table 1. PHASES data for V819 Herculis. All quantities are in the ICRS 2000.0 reference frame. The uncertainty values presented in this data have all been scaled by a factor of 2 over the formal (internal) uncertainties within each given night. Column $6, \phi_{\mathrm{e}}$, is the angle between the major axis of the uncertainty ellipse and the right ascension axis, measured from increasing differential right ascension through increasing differential declination (the position angle of the uncertainty ellipse's orientation is $90-\phi_{\mathrm{e}}$ ). The last column is the number of scans taken during a given night. The quadrant was chosen such that the larger fringe contrast is designated the primary (contrast is a combination of source luminosity and interferometric visibility).

\begin{tabular}{|c|c|c|c|c|c|c|c|c|c|}
\hline JD-2 400000.5 & $\begin{array}{l}\delta \mathrm{RA} \\
\text { (mas) }\end{array}$ & $\begin{array}{l}\delta \text { Dec } \\
\text { (mas) }\end{array}$ & $\begin{array}{l}\sigma_{\min } \\
(\mu \text { as })\end{array}$ & $\begin{array}{l}\sigma_{\text {maj }} \\
(\mu \text { as })\end{array}$ & $\begin{array}{l}\phi_{\mathrm{e}} \\
(\mathrm{deg})\end{array}$ & $\begin{array}{l}\sigma_{\mathrm{RA}} \\
(\mu \mathrm{as})\end{array}$ & $\begin{array}{l}\sigma_{\mathrm{Dec}} \\
(\mu \text { as })\end{array}$ & $\frac{\sigma_{\mathrm{RA}, \mathrm{Dec}}^{2}}{\sigma_{\mathrm{RA}} \sigma_{\mathrm{Dec}}}$ & $N$ \\
\hline 53109.4777819 & 49.6404 & -84.4971 & 14.7 & 566.0 & 158.77 & 527.6 & 205.4 & -0.99704 & 2011 \\
\hline 53110.4800679 & 48.0940 & -84.1339 & 23.7 & 1199.8 & 159.53 & 1124.1 & 420.3 & -0.99818 & 1334 \\
\hline 53123.4555720 & 49.1857 & -85.9322 & 36.2 & 1015.6 & 162.47 & 968.5 & 307.9 & -0.99239 & 1378 \\
\hline 53130.4397626 & 48.4773 & -86.4138 & 13.1 & 411.8 & 162.95 & 393.7 & 121.4 & -0.99359 & 2537 \\
\hline 53137.4279917 & 48.3926 & -87.1400 & 27.9 & 560.7 & 164.34 & 539.9 & 153.7 & -0.98203 & 1226 \\
\hline 53145.3928594 & 48.3081 & -87.8018 & 27.5 & 633.0 & 161.59 & 600.7 & 201.6 & -0.98962 & 1673 \\
\hline 53168.3368802 & 47.0273 & -89.7517 & 30.1 & 680.1 & 162.93 & 650.2 & 201.7 & -0.98777 & 1409 \\
\hline 53172.3496326 & 47.3437 & -90.1341 & 12.6 & 339.9 & 168.29 & 332.9 & 70.1 & -0.98310 & 2560 \\
\hline 53173.3294522 & 47.1580 & -90.3605 & 16.0 & 154.8 & 33.97 & 128.7 & 87.5 & 0.97549 & 2904 \\
\hline 53181.3314386 & 46.4330 & -90.7861 & 15.0 & 349.0 & 169.71 & 343.4 & 64.1 & -0.97112 & 2795 \\
\hline 53186.3020911 & 45.6581 & -91.1217 & 36.4 & 853.0 & 166.80 & 830.5 & 198.0 & -0.98202 & 706 \\
\hline 53187.3022539 & 46.1426 & -91.2229 & 26.0 & 882.1 & 166.94 & 859.3 & 201.0 & -0.99114 & 1578 \\
\hline 53197.2663645 & 46.1848 & -92.1526 & 9.5 & 234.1 & 164.87 & 226.0 & 61.8 & -0.98714 & 5218 \\
\hline 53198.2404599 & 46.2934 & -92.2558 & 11.4 & 109.3 & 160.36 & 103.0 & 38.3 & -0.94842 & 5404 \\
\hline 53199.2897673 & 44.0250 & -91.9450 & 49.2 & 3283.0 & 171.42 & 3246.2 & 492.3 & -0.99487 & 946 \\
\hline 53208.2505295 & 46.4281 & -92.4906 & 13.2 & 362.5 & 37.67 & 287.1 & 221.8 & 0.99719 & 6558 \\
\hline 53214.2391404 & 45.6333 & -93.3432 & 10.9 & 251.7 & 169.45 & 247.5 & 47.3 & -0.97195 & 5251 \\
\hline 53215.2293360 & 45.6361 & -93.5176 & 9.5 & 221.3 & 167.53 & 216.1 & 48.7 & -0.97963 & 5723 \\
\hline 53221.2207072 & 46.2536 & -92.9732 & 17.6 & 683.9 & 38.91 & 532.3 & 429.8 & 0.99861 & 3998 \\
\hline 53228.2083438 & 45.0879 & -94.3314 & 14.4 & 201.2 & 169.45 & 197.8 & 39.5 & -0.92815 & 3180 \\
\hline 53233.1820405 & 45.0989 & -94.8462 & 12.0 & 129.5 & 167.67 & 126.5 & 30.0 & -0.91190 & 3303 \\
\hline 53234.2006462 & 44.8264 & -94.7640 & 15.2 & 75.7 & 172.74 & 75.1 & 17.9 & -0.51361 & 3701 \\
\hline 53235.2168202 & 45.2148 & -94.9186 & 17.0 & 214.3 & 176.57 & 213.9 & 21.2 & -0.60018 & 2094 \\
\hline 53236.1665478 & 44.4594 & -94.8865 & 9.5 & 156.1 & 166.59 & 151.9 & 37.4 & -0.96553 & 6684 \\
\hline 53481.5043302 & 30.4429 & -103.3356 & 22.2 & 622.2 & 38.18 & 489.3 & 385.0 & 0.99730 & 3301 \\
\hline
\end{tabular}

The largest possible astrometric shift by a star spot is given by evaluating a slightly different model. In this case, the star spot fills the (non-circular) area from the star's edge to a chord at distance $x_{o}$ from the star's true center. The astrometric shift is

$$
\begin{aligned}
\frac{x_{c}}{R} & =\frac{\int_{-R}^{x_{o}} \int_{-\sqrt{R^{2}-x^{2}}}^{\sqrt{R^{2}-x^{2}}} x \mathrm{~d} y \mathrm{~d} x}{R \int_{-R}^{x_{o}} \int_{-\sqrt{R^{2}-x^{2}}}^{\sqrt{R^{2}}} \mathrm{~d} y \mathrm{~d} x} \\
& =-\frac{2\left(1-\frac{x_{o}^{2}}{R^{2}}\right)^{3 / 2}}{3\left(\frac{\pi}{2}+\arcsin \frac{x_{o}}{R}+\frac{x_{o}}{R}\left(1-\frac{x_{o}^{2}}{R^{2}}\right)^{1 / 2}\right)}
\end{aligned}
$$

with corresponding photometric variations of

$$
\frac{F}{F_{o}}=\frac{1}{\pi}\left(\frac{\pi}{2}+\arcsin \frac{x_{o}}{R}+\frac{x_{o}}{R}\left(1-\frac{x_{o}^{2}}{R^{2}}\right)^{1 / 2}\right) .
$$

In the V819 Herculis system, the effect of a single, cold starspot is maximally $\sim 25 \mu$ as assuming a stellar radius of 0.8 mas. If multiple starspots cause the variability, this effect is reduced.
In addition, the effect of limb-darkening is to further reduce the astrometric error.

\subsubsection{PHASES observations during $\mathrm{Ba}-\mathrm{Bb}$ eclipses}

Using the published sizes and temperatures for $\mathrm{Ba}$ and $\mathrm{Bb}$ from van Hamme et al. (1994), it is found that the magnitude of the astrometric shift in CL position during eclipse compared to what it would be outside of eclipse can be greater than $100 \mu$ as. Because this shift is larger than PHASES astrometric measurement precisions, six measurements taken during eclipse are omitted from the data tables and the fits.

\subsection{Previous differential astrometry measurements}

Previously published differential astrometry measurements of the A-B (wide) system have been tabulated by Hartkopf et al. (2004) in the Fourth Catalog of Interferometric Measurements of Binary Stars. In several cases discrepancies were found between uncertainties quoted in the original works (or uncertainty 


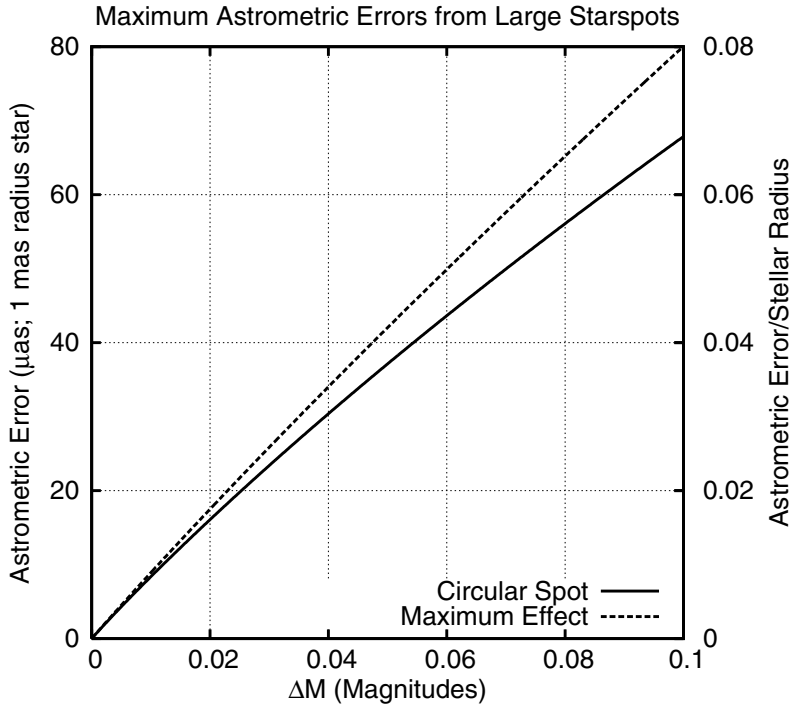

Fig. 1. The maximum effect of star spots on astrometric measurements versus the photometric variations they cause.

estimates omitted in the catalog); in these cases the uncertainty estimates from the original works are used. All of these measurements were made using the technique of speckle interferometry. These measurements are included in the combined fit to help complete coverage of the A-B orbit.

Many of the previous differential astrometry measurements were published without any associated uncertainties. To allow these to be used in combined fits with other data sets, the average uncertainties were determined as follows. The uncertainties were initially assigned values of 10 milli-arcseconds in separation and 1 degree in position angle. A (single) Keplerian model was fit to the data, and residuals in separation and position angle were treated individually to remove outliers and update the uncertainty estimates. This procedure was iterated until uncertainties were found consistent with the scatter. A double Keplerian model (as in Eq. (8), to allow for the Ba-Bb subsystem) does not improve the fit; the measurements are insensitive to this small signal. These 22 data points have average uncertainties of 5.92 milli-arcseconds and 0.689 degrees.

A Keplerian model was fit to the data points for which uncertainty estimates were available to determine whether these were systematically too large or too small, and to find outliers. The uncertainty estimates are found to be systematically too small; this factor was larger in position angle than in separation. Upon iteration, it was found that the separation uncertainties for these 12 data points needed to be increased by a factor of 2.43 and the position angle uncertainties by 3.53. Again no improvement was seen in fitting to a double Keplerian model.

These previously published measurements are listed in Tables 2 and 3.

\subsection{Radial velocity data}

A large number of radial velocity measurements of components $\mathrm{A}$ and $\mathrm{Ba}$ from four observatories were reported in Scarfe et al. (1994). Scarfe et al. indicate several measurements as outliers; these measurements have not been used.
Table 2. Previous differential astrometry data with published uncertainties for V819 Herculis. $\rho$ uncertainties have been increased by a factor of 2.43 and those for $\theta$ by a factor of 3.53. In many cases $\theta$ has been changed by 180 degrees from the value appearing in the original works.

\begin{tabular}{llllll}
\hline \hline Year & $\begin{array}{l}\rho \\
(\mathrm{mas})\end{array}$ & $\begin{array}{l}\sigma_{\rho} \\
(\mathrm{deg})\end{array}$ & $\begin{array}{l}\sigma_{\theta} \\
(\mathrm{mas})\end{array}$ & Reference \\
\hline 1980.4766 & 36 & 278.1 & 4.86 & 7.06 & McAlister et al. (1983) \\
1980.4820 & 47 & 265.7 & 4.86 & 7.06 & McAlister et al. (1983) \\
1982.2924 & 107 & 154.7 & 2.43 & 1.76 & Fu et al. (1997) \\
1991.3192 & 46 & 252.4 & 7.29 & 0.71 & Hartkopf et al. (1994) \\
1992.3104 & 48 & 123.4 & 7.29 & 0.71 & Hartkopf et al. (1994) \\
1992.6086 & 69 & 141.2 & 7.29 & 7.06 & Balega et al. (1999) \\
1993.2058 & 96 & 149.9 & 7.29 & 0.71 & Hartkopf et al. (1994) \\
1994.7078 & 102 & 169.8 & 1.49 & 1.06 & Hartkopf et al. (2000) \\
1995.3138 & 86 & 177.9 & 7.29 & 0.71 & Hartkopf et al. (1997) \\
1999.5681 & 115 & 164.0 & 6.90 & 3.21 & Horch et al. (2002) \\
1999.5681 & 109 & 160.4 & 6.90 & 3.21 & Horch et al. (2002) \\
2000.5563 & 101 & 175.6 & 6.90 & 3.21 & Horch et al. (2002) \\
\hline
\end{tabular}

There are 72 component A velocities and 50 component Ba velocities in the McDonald/Kitt Peak data set, 70 component A and 49 component $\mathrm{Ba}$ velocities in the DAO set, and 92 component $\mathrm{A}$ and 90 component Ba velocities in the DDO data set.

The velocity measurements for each of three data sets presented were fit to double Keplerian models separately to determine the average velocity uncertainties (measurements from McDonald and Kitt Peak were mixed together in the original work, and these were analyzed together as one group). As noted in Scarfe et al. (1994), the velocity precisions for component A differed from those of Ba. Uncertainties were derived for each data set by fitting to a double Keplerian model and examining the scatter in the residuals for each component separately. The uncertainty guesses were updated and the procedure iterated. The initial values for the component A velocities were $0.46 \mathrm{~km} \mathrm{~s}^{-1}$ for the McDonald/Kitt Peak and DAO data sets, and $0.92 \mathrm{~km} \mathrm{~s}^{-1}$ for the DDO set; for component $\mathrm{Ba}$, all were set to $2 \mathrm{~km} \mathrm{~s}^{-1}$. The average A and $\mathrm{Ba}$ uncertainties are $0.43 \mathrm{~km} \mathrm{~s}^{-1}$ and $1.955 \mathrm{~km} \mathrm{~s}^{-1}$ for the McDonald/Kitt Peak velocities, $0.465 \mathrm{~km} \mathrm{~s}^{-1}$ and $3.025 \mathrm{~km} \mathrm{~s}^{-1}$ for the DAO measurements, and $1.015 \mathrm{~km} \mathrm{~s}^{-1}$ and $3.105 \mathrm{~km} \mathrm{~s}^{-1}$ for the DDO observations.

\section{Orbital models}

Basic models have been applied to the astrometric data. The simplifying assumption was made that the $\mathrm{Ba}-\mathrm{Bb}$ subsystem is unperturbed by star A over the timescale of the observing program, allowing the model to be split into a wide (slow) interaction between star $\mathrm{A}$ and the center of mass $(\mathrm{CM})$ of $\mathrm{B}$, and the close (fast) interaction between stars $\mathrm{Ba}$ and $\mathrm{Bb}$. The results presented in this paper result from modeling both the A-B and $\mathrm{Ba}-\mathrm{Bb}$ motions with Keplerian orbits.

In general, one cannot simply superimpose the results of the two orbits. The observable in the PHASES measurements is the separation of star A and the CL of the Ba-Bb subsystem. 
Table 3. Previous interferometric differential astrometry data without published uncertainties for V819 Herculis. The uncertainties presented in this table were determined by the scatter in the data. For the values in this table, all uncertainties were taken to be $\sigma_{\rho}=5.92$ mas and $\sigma_{\theta}=$ 0.689 degrees.

\begin{tabular}{llll|llll}
\hline \hline Year & $\rho$ & $\theta$ & Reference & Year & $\begin{array}{l}\rho \\
(\mathrm{mas})\end{array}$ & $\begin{array}{l}\theta \\
(\mathrm{deg})\end{array}$ & Reference \\
\hline 1981.4568 & 0.065 & 134.1 & McAlister et al. (1984) & 1984.7009 & 0.076 & 188.3 & McAlister et al. (1987a) \\
1981.4678 & 0.061 & 134.2 & McAlister et al. (1984) & 1985.4816 & 0.051 & 224.4 & McAlister et al. (1987a) \\
1981.4706 & 0.063 & 135.2 & McAlister et al. (1984) & 1985.5228 & 0.047 & 228.3 & McAlister et al. (1987b) \\
1981.4732 & 0.062 & 135.6 & McAlister et al. (1984) & 1986.4079 & 0.034 & 354.9 & Balega et al. (1989) \\
1982.5027 & 0.109 & 155.2 & McAlister et al. (1987a) & 1986.6454 & 0.038 & 109.0 & Balega et al. (1989) \\
1983.0702 & 0.112 & 163.0 & McAlister et al. (1987a) & 1987.2673 & 0.080 & 140.9 & McAlister et al. (1989) \\
1983.4202 & 0.105 & 165.8 & McAlister et al. (1987a) & 1988.2529 & 0.107 & 157.5 & McAlister et al. (1989) \\
1983.7151 & 0.107 & 171.3 & McAlister et al. (1987a) & 1988.6599 & 0.108 & 162.4 & McAlister et al. (1990) \\
1984.3732 & 0.100 & 180.2 & McAlister et al. (1987a) & 1989.2305 & 0.101 & 170.1 & McAlister et al. (1990) \\
1984.3760 & 0.088 & 181.4 & McAlister et al. (1987a) & 1989.7058 & 0.091 & 177.7 & Hartkopf et al. (1992) \\
1984.3840 & 0.087 & 181.5 & McAlister et al. (1987a) & 1990.2651 & 0.074 & 190.2 & Hartkopf et al. (1992) \\
\hline
\end{tabular}

Because the $\mathrm{CL}$ of $\mathrm{Ba}-\mathrm{Bb}$, the $\mathrm{CM}$ of $\mathrm{Ba}-\mathrm{Bb}$, and the location of star Ba are generally all unequal, a coupling amplitude must be added to the combined model. This coupling amplitude measures the relative size of the semi-major axis of the $\mathrm{Ba}-\mathrm{Bb}$ subsystem to that of the motion of the CL of the $\mathrm{Ba}-\mathrm{Bb}$ subsystem. The sign of the superposition is determined by the relative sizes of the mass and luminosity ratios of the stars $\mathrm{Ba}$ and $\mathrm{Bb}$. As an example, if the CL is located between the CM of $\mathrm{Ba}-\mathrm{Bb}$ and the location of star $\mathrm{Ba}$, the motion of the $\mathrm{CL}$ will be in opposite direction to the vector pointing from $\mathrm{Ba}$ to $\mathrm{Bb}$. For a subsystem with mass ratio $M_{\mathrm{Bb}} / M_{\mathrm{Ba}}$ and luminosity ratio $L_{\mathrm{Bb}} / L_{\mathrm{Ba}}$, the observed quantity is

$\overrightarrow{y_{\mathrm{obs}}}=\overrightarrow{r_{\mathrm{A}-\mathrm{B}}}-\frac{M_{\mathrm{Bb}} / M_{\mathrm{Ba}}-L_{\mathrm{Bb}} / L_{\mathrm{Ba}}}{\left(1+M_{\mathrm{Bb}} / M_{\mathrm{Ba}}\right)\left(1+L_{\mathrm{Bb}} / L_{\mathrm{Ba}}\right)} \overrightarrow{r_{\mathrm{Ba}-\mathrm{Bb}}}$

where $\overrightarrow{r_{\mathrm{A}-\mathrm{B}}}$ is the model separation pointing from star $\mathrm{A}$ to the $\mathrm{CM}$ of $\mathrm{B}$, and $\overrightarrow{\mathrm{Ba}-\mathrm{Bb}}$ is the model separation pointing from star $\mathrm{Ba}$ to star $\mathrm{Bb}$. Including this coupling term for astrometric data is important when a full analysis including radial velocity data is made.

Alternatively, one can directly combine a model of the A-B system with a model of the motion of the CL of Ba-Bb. For purely astrometric data such a model is appropriate. In this case, there is no sign change for the Ba-Bb CL model, and no extra coupling amplitude is required. This solution can be used to provide a high-precision apparent orbit which can be used to compare different instruments. This model differs from the previous one in that the true semi-major axis of the $\mathrm{Ba}-\mathrm{Bb}$ orbit is not a parameter, but is replaced by the apparent photocentric motion directly.

$\overrightarrow{y_{\mathrm{obs}}}=\overrightarrow{r_{\mathrm{A}-\mathrm{B}}}+\overrightarrow{r_{\mathrm{Ba}-\mathrm{Bb}, \text { C.O.L. }}}$

\section{Orbital solution and derived quantities}

The best-fit combined astrometry-radial velocity orbital solution produces a set of parameters listed in Table 4 . The reduced $\chi_{r}^{2}$ of the combined fit to PHASES, radial velocity, and previous differential astrometry data is 1.33 . This combined set
Table 4. Orbit models for V819 Herculis. Pre.: previous differential astrometry measurements, listed in Tables 2 and 3. The luminosity ratio $L_{\mathrm{Bb}} / L_{\mathrm{Ba}}$ is for $K$-band observations.

\begin{tabular}{ll}
\hline \hline & PHASES \\
& + Pre. $+\mathrm{RV}$ \\
\hline$P_{\mathrm{A}-\mathrm{B}}($ days $)$ & $2019.79 \pm 0.36$ \\
$T_{0, \mathrm{~A}-\mathrm{B}}(\mathrm{MJD})$ & $52628.1 \pm 1.3$ \\
$e_{\mathrm{A}-\mathrm{B}}$ & $0.6731 \pm 0.0015$ \\
$i_{\mathrm{A}-\mathrm{B}}($ degrees $)$ & $57.09 \pm 0.22$ \\
$\omega_{\mathrm{A}-\mathrm{B}}($ degrees $)$ & $42.55 \pm 0.23$ \\
$\Omega_{\mathrm{A}-\mathrm{B}}($ degrees $)$ & $322.40 \pm 0.16$ \\
$P_{\mathrm{Ba}-\mathrm{Bb}}($ days $)$ & $2.2296337 \pm 1.9 \times 10^{-6}$ \\
$T_{0, \mathrm{Ba}-\mathrm{Bb}}(\mathrm{MJD})$ & $52627.18 \pm 0.30$ \\
$e_{\mathrm{Ba}-\mathrm{Bb}}$ & $0.0041 \pm 0.0033$ \\
$i_{\mathrm{Ba}-\mathrm{Bb}}($ degrees $)$ & $79.0 \pm 3.3$ \\
$\omega_{\mathrm{Ba}-\mathrm{Bb}}($ degrees $)$ & $47 \pm 48$ \\
$\Omega_{\mathrm{Ba}-\mathrm{Bb}}($ degrees $)$ & $312.9 \pm 4.8$ \\
$V_{0, M / K}\left(\mathrm{~km} \mathrm{~s}^{-1}\right)$ & $-3.388 \pm 0.059$ \\
$V_{0, \mathrm{DAO}}\left(\mathrm{km} \mathrm{s}^{-1}\right)$ & $-3.373 \pm 0.064$ \\
$V_{0, \mathrm{DDO}}\left(\mathrm{km} \mathrm{s}^{-1}\right)$ & $-3.35 \pm 0.12$ \\
$M_{\mathrm{A}}\left(M_{\odot}\right)$ & $1.765 \pm 0.095$ \\
$M_{\mathrm{Ba}+\mathrm{Bb}}\left(M_{\odot}\right)$ & $2.512 \pm 0.067$ \\
$M_{\mathrm{Bb}} / M_{\mathrm{Ba}}$ & $0.757 \pm 0.020$ \\
$L_{\mathrm{Bb}} / L_{\mathrm{Ba}}$ & $0.261 \pm 0.045$ \\
$d(\mathrm{parsecs})$ & $67.96 \pm 0.87$ \\
\hline &
\end{tabular}

has 521 degrees of freedom with 20 parameters. This value for $\chi_{r}^{2}$ is slightly higher than one would expect, but this is likely due to the manner in which the uncertainties had to be derived. All parameter uncertainties have been increased by a factor of $\sqrt{1.33}$ to reflect this difference. It is noted that the value for the inclination of the $\mathrm{Ba}-\mathrm{Bb}$ pair of $79.0 \pm 3.3$ degrees agrees with that determined by eclipse observations $(81.00 \pm 0.36$ for the synchronous and $80.63 \pm 0.33$ for the asynchronous solutions of van Hamme et al. 1994). Also presented is a table of derived parameters of direct astrophysical interest (5). 

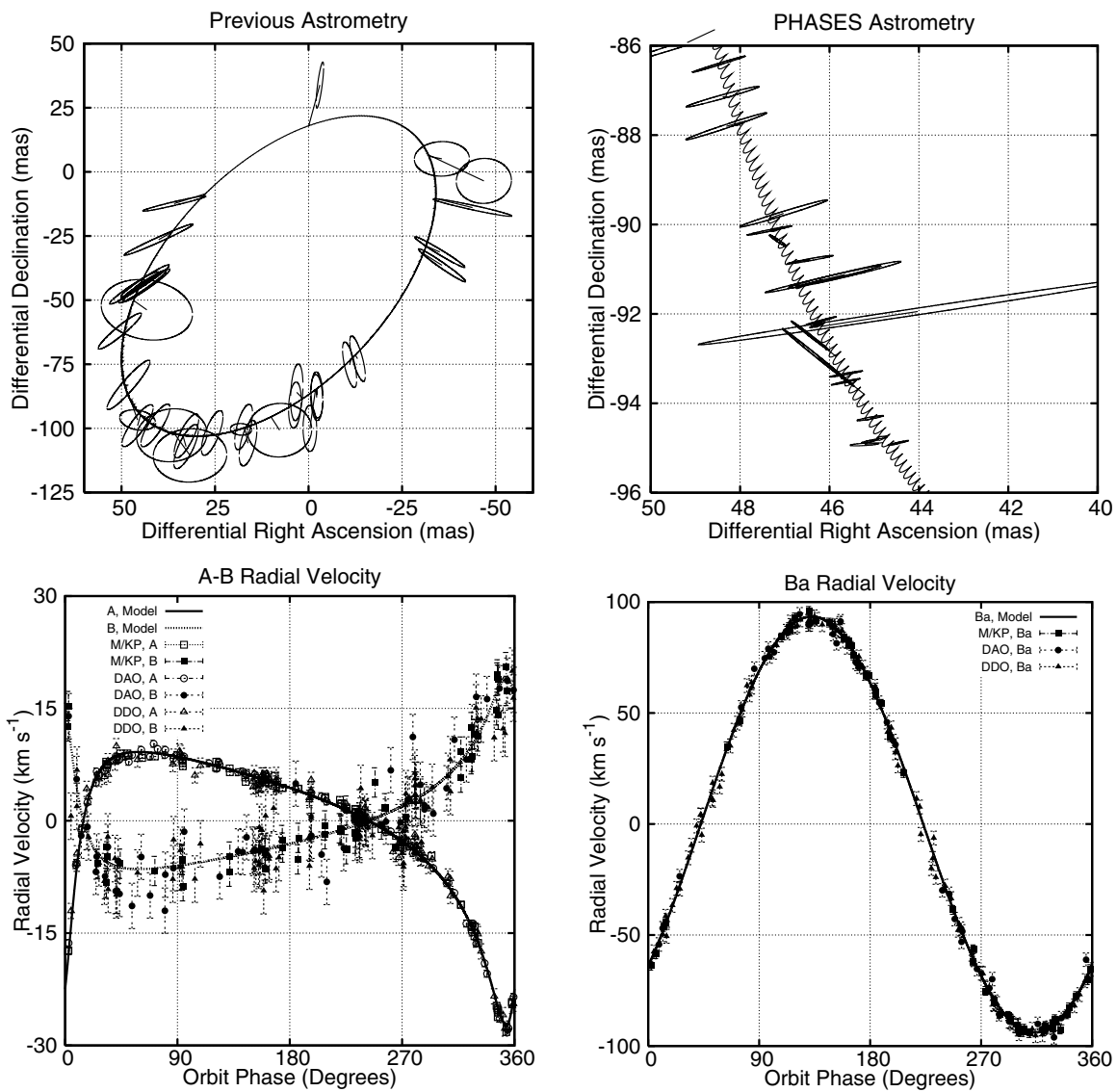

Fig. 2. The orbit of V819 Herculis. (Top left) Previous (speckle) differential astrometry measurements with derived uncertainty ellipses. (Top right) One season of PHASES astrometry. (Bottom left) CM velocities of the wide pair. (Bottom right) Radial velocity of the Ba component. The A-B motion has been removed for clarity.

Table 5. Physical parameters for V819 Herculis derived from the combined orbital solution.

\begin{tabular}{ll}
\hline \hline Parameter & Derived Value \\
\hline$a_{\mathrm{A}-\mathrm{B}}(\mathrm{AU})$ & $5.076 \pm 0.046$ \\
$a_{\mathrm{Ba}-\mathrm{Bb}}(\mathrm{AU})$ & $0.04541 \pm 0.00040$ \\
$\cos \Phi($ degrees $)$ & $23.6 \pm 4.9$ \\
$M_{\mathrm{Ba}}\left(M_{\odot}\right)$ & $1.430 \pm 0.041$ \\
$M_{\mathrm{Bb}}\left(M_{\odot}\right)$ & $1.082 \pm 0.033$ \\
\hline
\end{tabular}

A fit to the astrometric data alone does not constrain several of the orbital parameters, but does constrain apparent semi-major axes of the wide and narrow orbits to good precision: $a_{\mathrm{A}-\mathrm{B}}=$ $73.9 \pm 0.6$ mas and $a_{\mathrm{Ba}-\mathrm{Bb}}=108 \pm 9 \mu$ as (Ba-Bb CL orbit).

The combined fit $\mathrm{A}-\mathrm{Ba}-\mathrm{Bb}$ orbit is plotted is Fig. 2; PHASES measurements of the $\mathrm{Ba}-\mathrm{Bb}$ orbit CL motion is plotted in Fig. 3. Residuals to the combined fit are shown in Figs. 4-6; no evidence for additional system components is observed.

\subsection{Mutual inclination}

The mutual inclination $\Phi$ of two orbits is given by

$\cos \Phi=\cos i_{1} \cos i_{2}+\sin i_{1} \sin i_{2} \cos \left(\Omega_{1}-\Omega_{2}\right)$ where $i_{1}$ and $i_{2}$ are the orbital inclinations and $\Omega_{1}$ and $\Omega_{2}$ are the longitudes of the ascending nodes. From the combined orbit solution a value for this angle of $23.6 \pm 4.9$ degrees is derived for the V819 Her system. This low value is below the limit for inclination-eccentricity oscillations derived by Kozai (1962; 39.2 degrees), and is consistent with the small measured value of the eccentricity of the $\mathrm{Ba}-\mathrm{Bb}$ pair.

The mutual inclination of the orbits of triple systems is of particular interest for understanding the conditions under which the system formed (Sterzik \& Tokovinin 2002). Without both radial velocity and visual (or astrometric) orbits for both systems in a triple, unambiguous determinations of the longitudes of the ascending nodes (and thus of the mutual inclination) are impossible. To date there has only been a very small number of cases where the mutual inclination can be unambiguously determined (Table 6).

With the tally of systems for which unambiguous mutual inclinations have been determined now at six, it is reasonable to consider the distribution of these orientations. The previous work on this subject is that of Sterzik \& Tokovinin (2002), who determined theoretical distributions resulting from a variety of initial conditions within star forming regions. At the time, the authors cited only three systems from which mutual inclinations were known, listed in a previous work by one of them (Tokovinin 1993). (One of these three, $\zeta$ Cnc, is most recently listed by Heintz (1996) as still having an ambiguous 

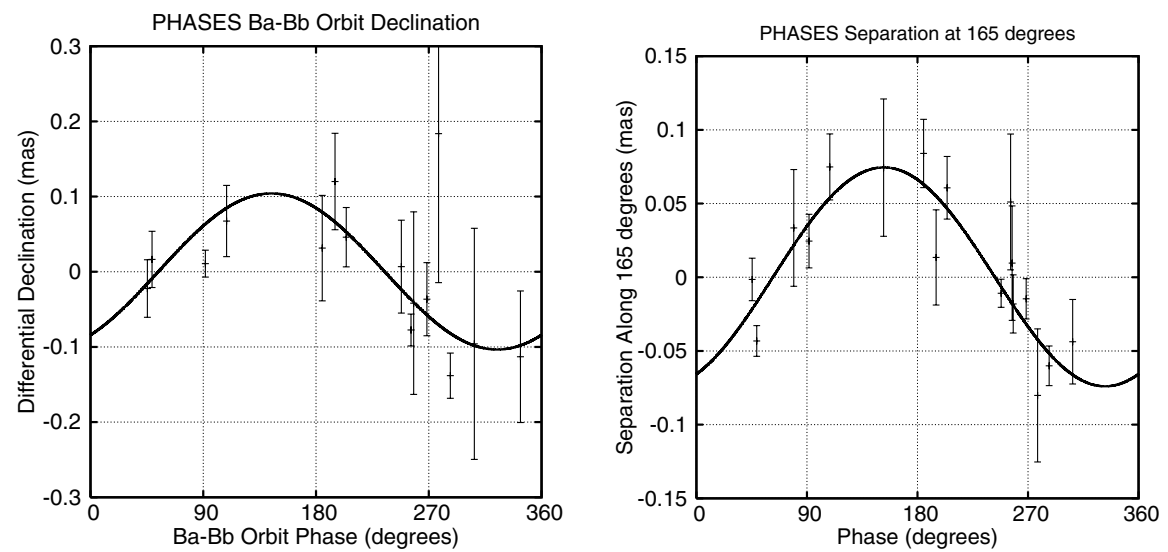

Fig. 3. CL astrometric motion of the V819 Herculis Ba-Bb system, as measured by PHASES observations along the declination axis (left) and along an axis rotated 165 degrees East of North (equivalent to position angle 285 degrees; right); this is the median position angle of the minor axis of the PHASES uncertainty ellipses. Because the orientation of the PHASES uncertainty ellipses varies from night to night, no single axis is ideal for exhibiting the PHASES precisions, but this median axis is best aligned to do so. The wide A-B orbital motion has been removed for both plots. The error bars plotted have been stretched by a factor of 2 over the formal uncertainties as discussed in the text. The high ellipticity of the uncertainty ellipses causes neither the right ascension nor the declination uncertainties to be near the precision of the minor axis uncertainties, which have median uncertainty of $15.2 \mu$ as. For clarity, measurements with projected uncertainties larger than $200 \mu$ as are not shown in the plots. Zero phase indicates periastron passage.
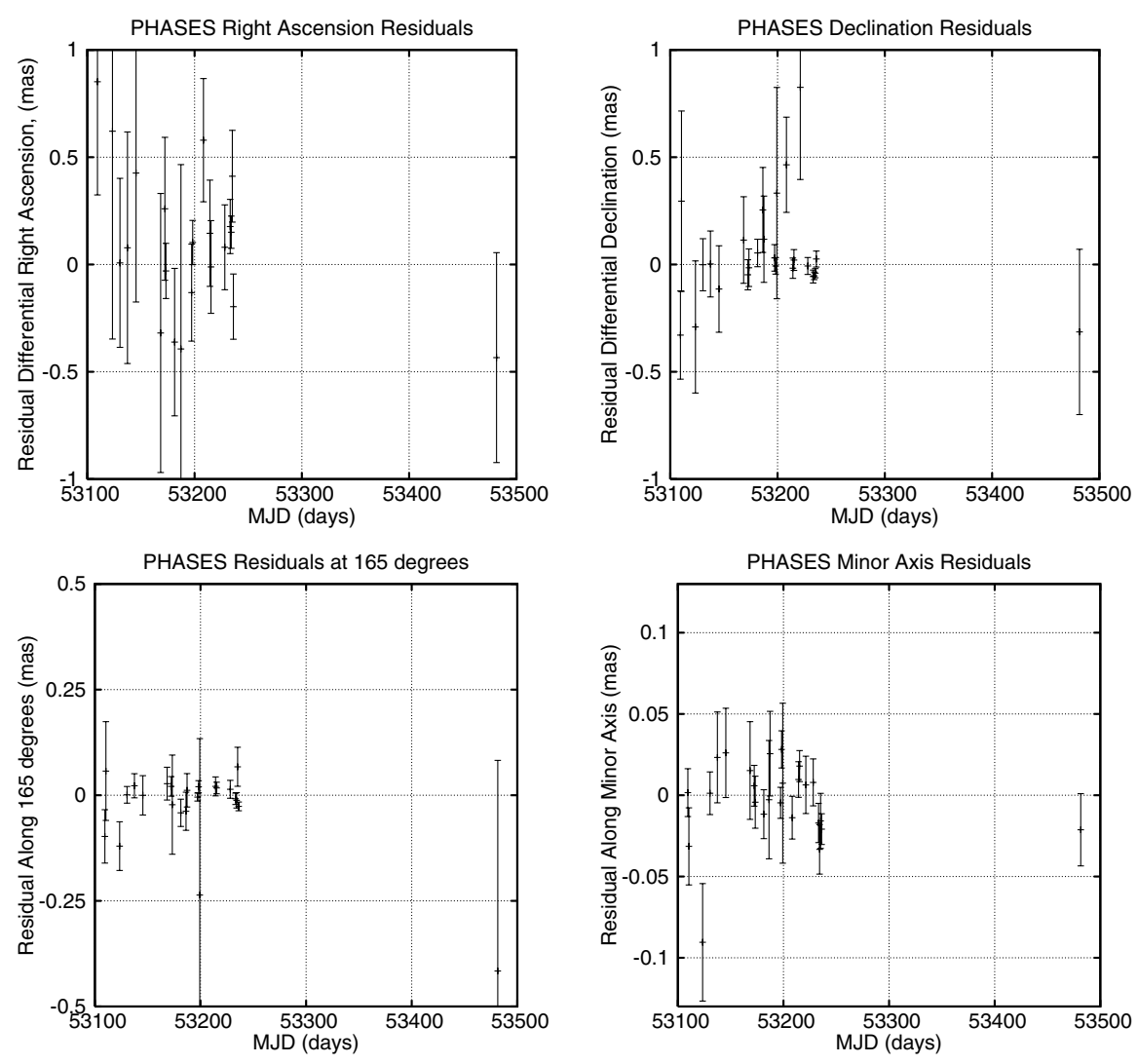

Fig. 4. Residuals for PHASES differential astrometry of V819 Herculis. The error bars plotted have been stretched by a factor of 2 over the formal uncertainties as discussed in the text. The high ellipticity of the uncertainty ellipses causes neither the right ascension nor the declination uncertainties to be near the precision of the minor axis uncertainties, which have median uncertainty of $15.2 \mu$ as. Due to the roughly North-South alignment of the baseline used for most of the measurements, the more sensitive axis was typically declination. The right ascension and declination plots show only those points for which the projected error bar is less than 1 milli-arcsecond. The bottom left plot shows the residuals along a direction that is 165 degrees from increasing differential right ascension through increasing differential declination (equivalent to position angle 285 degrees), which corresponds to the median direction of the minor axis of the PHASES uncertainty ellipses; only measurements with uncertainties less than $500 \mu$ as along this axis are plotted. Because the orientation of the PHASES uncertainty ellipses varies from night to night, no single axis is ideal for exhibiting the PHASES precisions, but this median axis is best aligned to do so. The bottom right plot shows residuals along the minor axis of each measurement's uncertainty ellipse. 

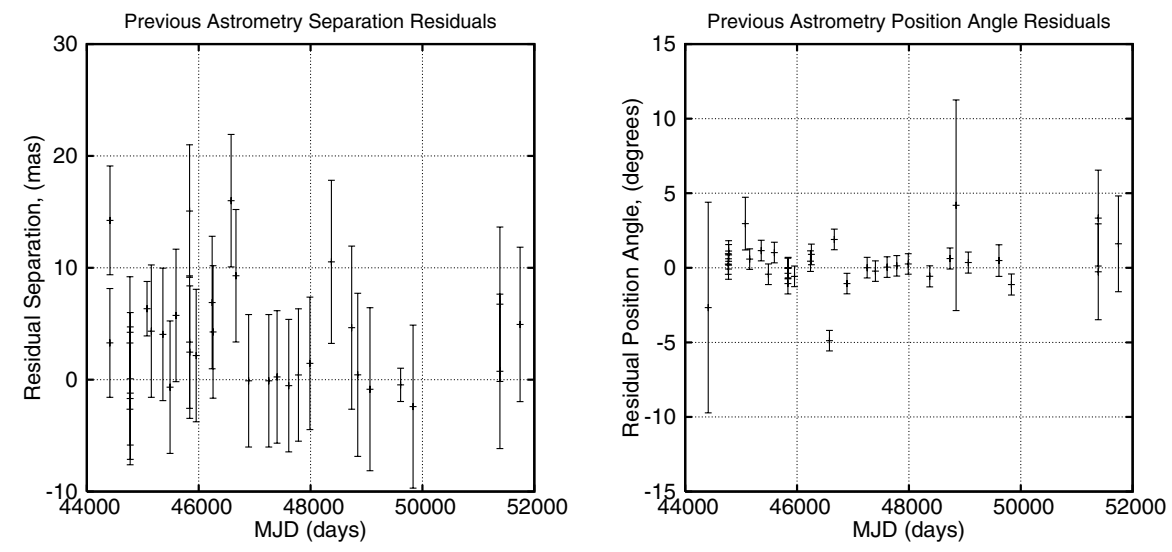

Fig. 5. Residuals to the combined model for previous differential astrometry of V819 Herculis.
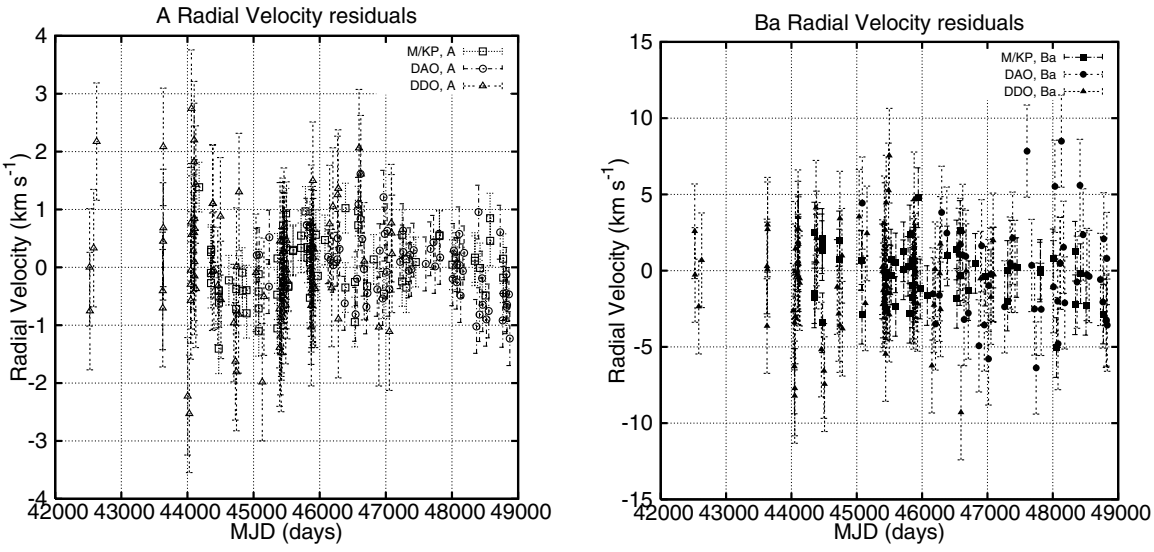

Fig. 6. Residuals to the combined model for radial velocimetry of V819 Herculis (Scarfe et al. 1994).

Table 6. Unambiguously known mutual inclinations of triple systems. The value for Algol is determined using the measurement precisions and values of Pan et al. (1993) for all but the A-B nodal position angle of $52 \pm 5$ degrees from Lestrade et al. (1993).

\begin{tabular}{lll}
\hline \hline Star & $\begin{array}{l}\text { Mutual Inclination } \\
\text { (degrees) }\end{array}$ & Reference \\
\hline V819 Her & $23.6 \pm 4.9$ & This paper \\
$\kappa$ Peg & $43.8 \pm 3.0$ & (Muterspaugh et al. 2005) \\
$\eta$ Vir & $30.8 \pm 1.3$ & (Hummel et al. 2003) \\
$\epsilon$ Hya & 39.4 & (Heintz 1996) \\
$\xi$ UMa & 132.1 & (Heintz 1996) \\
Algol & $98.8 \pm 4.9$ & (Lestrade et al. 1993) \\
& & (Pan et al. 1993) \\
\hline
\end{tabular}

mutual inclination, and is not included here.) For comparison to real stars, Sterzik \& Tokovinin instead included the 22 triple systems in the Multiple Star Catalog (Tokovinin 1997) for which both visual orbits were known, but the ascending nodes had 180 degree ambiguities. To correct for this lack of information, for each system they included both possible mutual inclinations in a combined cumulative distribution (this distribution is referred to as ST). This procedure assumes the ambiguity is divided evenly between the lower and higher possible angles; i.e. that an equal number of the "true" mutual inclinations are the lesser of the two possible angles as are the greater.

In Fig. 7 the continuous distribution function of mutual inclinations for the six unambiguous systems (this distribution is referred to as $6 \mathrm{U}$ ) is plotted with ST and the theoretical distribution for random orientations (referred to simply as Random).

The two-sided Kolmogorov-Smirnov (K-S) probability for agreement between $6 \mathrm{U}$ and ST is 0.46 ; the one-sided KS probability between ST and Random is 0.07 and that for $6 \mathrm{U}$ and Random is 0.04 . Thus, the $6 \mathrm{U}$ set confirms the result of Sterzik $\&$ Tokovinin (2002) that mutual inclinations are not consistent with random orientations and show a slight preference for coplanarity. The sets $6 \mathrm{U}$ and ST agree much better with each other than either do with random orientations, but the agreement probability is still low; this is likely due to the assumption inherent to forming the ST set by including both possible orientations, which dilutes the distribution away from coplanarity. A greater number of systems is required to better determine the distributions, and observational selection effects should also be considered.

\section{Conclusions}

PHASES interferometric astrometry has been used to measure the orbital parameters of the triple star system V819 Herculis, and in particular to resolve the apparent orbital motion of the 


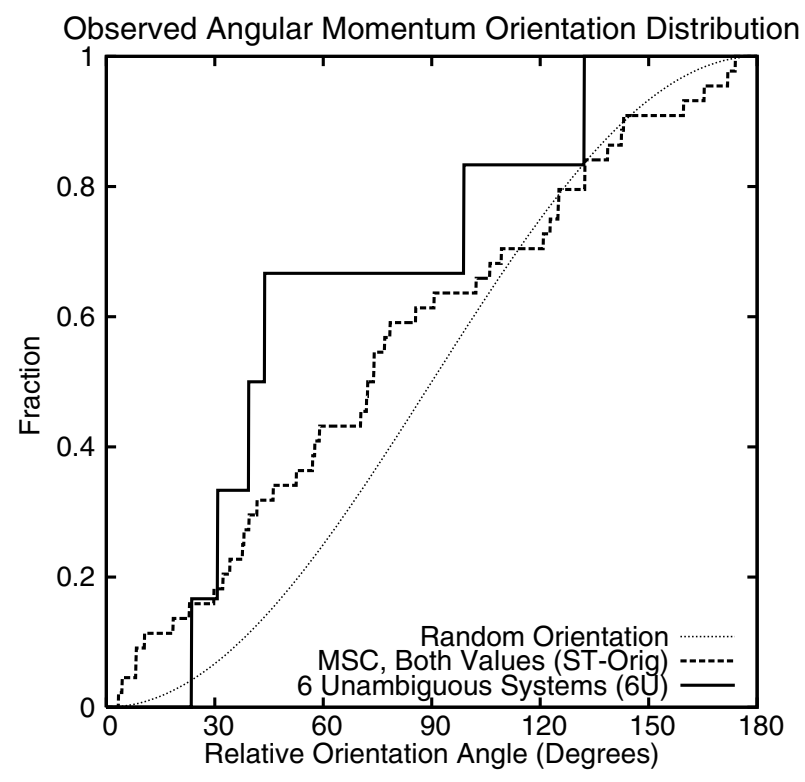

Fig. 7. Cumulative distribution of the observed distribution of angles between angular momentum vectors of the six systems for which unambiguous mutual inclinations have been determined. This is compared with the results from Sterzik \& Tokovinin (2002), who included 22 systems for which the mutual inclinations could only be determined ambiguously-two degenerate angles were both possible solutions due a 180 degree ambiguity in the longitude of the ascending node of at least one component of the triple system. Sterzik \& Tokovinin included both possible angles in their distribution. Also shown is the theoretical distribution for random orientations.

close $\mathrm{Ba}-\mathrm{Bb}$ pair. The amplitude of the $\mathrm{Ba}-\mathrm{Bb} \mathrm{CL}$ motion is only $108 \pm 9 \mu$ as, indicating the level of astrometric precision attainable with interferometric astrometry. By measuring both orbits one is able to determine the mutual inclination of the two orbits, which is found to be $23.6 \pm 4.9$ degrees. Such a low mutual inclination implies a lack of Kozai oscillations.

Further improvement in determining the system distance and component masses will require improved radial velocity data. Given that the A component is evolved and the $\mathrm{Ba}-\mathrm{Bb}$ system undergoes eclipses and hence the $\mathrm{B}$ components have accurately measured radii, this system may become a very fruitful laboratory for high-precision testing of stellar models.

Acknowledgements. We thank the support of the PTI collaboration, whose members have contributed designing an extremely reliable instrument for obtaining precision astrometric measurements. We acknowledge the extraordinary efforts of K. Rykoski, whose work in operating and maintaining PTI is invaluable and goes far beyond the call of duty. Part of the work described in this paper was performed at the Jet Propulsion Laboratory under contract with the National Aeronautics and Space Administration. Interferometer data was obtained at the Palomar Observatory using the NASA Palomar Testbed Interferometer, supported by NASA contracts to the Jet Propulsion Laboratory. We thank C. Scarfe for providing machinereadable tables of the radial velocity measurements. This research has made use of the Washington Double Star Catalog maintained at the U.S. Naval Observatory. This research has made use of the Simbad database, operated at CDS, Strasbourg, France. MWM acknowledges the support of the Michelson Graduate Fellowship program. BFL acknowledges support from a Pappalardo Fellowship in
Physics. MK is supported by the NASA grant NNG04GM62G and the Polish MSIST grant 1-P03D-021-29. PHASES is funded in part by the California Institute of Technology Astronomy Department, and by the National Aeronautics and Space Administration under Grant No. NNG05GJ58G issued through the Terrestrial Planet Finder Foundation Science Program.

\section{References}

Balega, I. I., Balega, Y. Y., \& Vasyuk, V. A. 1989, Speckle Interferometry Measurements of Binaries Using the 6-m Telescope in 1986, Bull. Special Astrof. OBS. North Caucasus 28, 102

Balega, I. I., Balega, Y. Y., Maksimov, A. F., et al. 1999, A\&AS, 140, 287

Colavita, M. M. 1994, A\&A, 283, 1027

Colavita, M. M., Wallace, J. K., Hines, B. E., et al. 1999, ApJ, 510, 505

Dempsey, R. C., Linsky, J. L., Fleming, T. A., \& Schmitt, J. H. M. M. 1993, ApJS, 86, 599

Drake, S. A., Simon, T., \& Linsky, J. L. 1989, ApJS, 71, 905

Fu, H., Hartkopf, W. I., Mason, B. D., et al. 1997, AJ, 114, 1623

Hartkopf, W. I., McAlister, H. A., \& Franz, O. G. 1992, AJ, 104, 810

Hartkopf, W. I., McAlister, H. A., Mason, B. D., et al. 1994, AJ, 108, 2299

Hartkopf, W. I., McAlister, H. A., Mason, B. D., et al. 1997, AJ, 114, 1639

Hartkopf, W. I., Mason, B. D., McAlister, H. A., et al. 2000, AJ, 119, 3084

Hartkopf, W. I., Mason, B. D., Wycoff, G. L., \& McAlister, H. A. 2004, Fourth catalog of interferometric measurements of binary stars, http://ad.usno.navy.mil/wds/int4.html

Heintz, W. D. 1996, AJ, 111, 408

Horch, E. P., Robinson, S. E., Meyer, R. D., et al. 2002, AJ, 123, 3442

Hummel, C. A., Benson, J. A., Hutter, D. J., et al. 2003, AJ, 125, 2630

Kaplan, G. H., Hughes, J. A., Seidelmann, P. K., Smith, C. A., \& Yallop, B. D. 1989, AJ, 97, 1197

Lane, B. F., \& Muterspaugh, M. W. 2004, ApJ, 601, 1129

Lestrade, J., Phillips, R. B., Hodges, M. W., \& Preston, R. A. 1993, ApJ, 410, 808

McAlister, H., Hartkopf, W. I., \& Franz, O. G. 1990, AJ, 99, 965

McAlister, H. A., Hartkopf, W. I., Hendry, E. M., Campbell, B. G., \& Fekel, F. C. 1983, ApJS, 51, 309

McAlister, H. A., Hartkopf, W. I., Gaston, B. J., Hendry, E. M., \& Fekel, F. C. 1984, ApJS, 54, 251

McAlister, H. A., Hartkopf, W. I., Hutter, D. J., \& Franz, O. G. 1987a, AJ, 93, 688

McAlister, H. A., Hartkopf, W. I., Hutter, D. J., Shara, M. M., \& Franz, O. G. 1987b, AJ, 93, 183

McAlister, H. A., Hartkopf, W. I., Sowell, J. R., Dombrowski, E. G., \& Franz, O. G. 1989, AJ, 97, 510

Muterspaugh, M. W., Lane, B. F., Konacki, M., et al. 2005, ApJ, submitted

Pan, X., Shao, M., \& Colavita, M. M. 1993, ApJ, 413, L129

Scarfe, C. D., Barlow, D. J., Fekel, F. C., et al. 1994, AJ, 107, 1529

Sterzik M. F., \& Tokovinin, A. A. 2002, A\&A, 384, 1030

Strassmeier, K. G., Hall, D. S., Fekel, F. C., \& Scheck, M. 1993, A\&AS, 100, 173

Tokovinin, A. A. 1993, Astron. Lett., 19, 383

Tokovinin, A. A. 1997, A\&AS, 124, 75

van Hamme, W. V., Hall, D. S., Hargrove, A. W., et al. 1994, AJ, 107, 1521

Wasson, R., Hall, D. S., Hargrove, A. W., et al. 1994, AJ, 107, 1514 